Article

\title{
The Zahl-Anzahl Distinction in Gottlob Frege: Arithmetic of Natural Numbers with Anzahl as a Primitive Term
}

\section{Eugeniusz Wojciechowski}

Division of Philosophy of Nature, Hugo Kołłataj Agriculture University of Cracow, 29 Listopada 46, 31-425 Cracow, Poland; eugeniusz.wojciechowski01@gmail.com

Received: 15 October 2019; Accepted: 24 December 2019; Published: 31 December 2019

check for updates

\begin{abstract}
The starting point is Peano's expression of the axiomatics of natural numbers in the framework of Leśniewski's elementary ontology. The author enriches elementary ontology with the so-called Frege's predication scheme and goes on to propose the formulations of this axiomatic, in which the original natural number $(N)$ term is replaced by the term Anzahl $(A)$. The functor of the successor $(S)$ is defined in it.
\end{abstract}

Keywords: Peano's axiomatics of natural numbers; Leśniewski's elementary ontology; Frege's predication scheme; Frege's Zahl-Anzahl distinction

\section{Introduction}

The term Anzahl functions in German in various numerical phrases, more or less defined. It appears in such contexts as "Anzahl der Äpfel" (i.e., "number of apples") or "neun ist Anzahl der Planeten des Sonnensystems" (i.e., "nine is the number of planets of the Solar System"). In numerical phrases of the indefinite type, we use the combination of the number name (Zahl), which is an abstract object, and the general name, which is the product of a general name (here, the word apple/apples) with a name present only implicitly, which somehow characterizes the objects falling within the extension of the first name (usually a general name referring to the place they occupy). However, number phrases of the definite type (v. gr. "neun ist Anzahl den Planeten des Sonnensystem"or "Anzahl... ist gleich dem Anzahl...") are discussed in Gottlob Frege's works. Number phrases of the definite type are

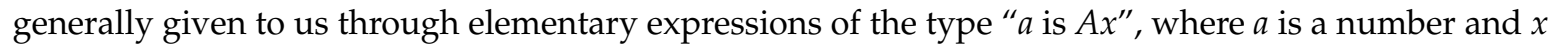
a (definite) general name. There is a specific functor $A$ (from "Anzahl") of the $n / n$ category. In Frege, "Anzahl" refers to a concept determined by a single-argument predicate (Begriffswort). Given these remarks, we enrich the framework of elementary ontology with the so-called Frege's predication scheme, to propose the formulations of these axiomatics in which the original natural number $(N)$ term is replaced by the term Anzahl $(A)$, and we present an original depiction of the arithmetic of natural numbers with $A n z a h l$ as a primitive term.

\section{Preliminaries}

\subsection{Elementary Ontology}

The specific axiom of elementary ontology (OE) is:

A0 $\quad x \varepsilon y \leftrightarrow \Sigma z(z \varepsilon x) \wedge \Pi z u(z \varepsilon x \wedge u \varepsilon x \rightarrow z \varepsilon u) \wedge \Pi z(z \varepsilon x \rightarrow z \varepsilon y)$

The secondary rules, which are direct consequences of axiom A0 include: 


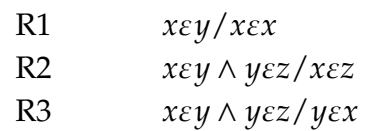

The nominal constants of object and contradictory object are defined as follows:

\begin{tabular}{|c|c|c|}
\hline DV & $x \varepsilon \mathrm{V} \leftrightarrow x \varepsilon x$ & \\
\hline $\mathrm{D} \Lambda$ & $x \varepsilon \Lambda \leftrightarrow x \varepsilon x \wedge \sim x \varepsilon x$ & \\
\hline
\end{tabular}

The functors of existence, singularity, being an object, weak inclusion, strong inclusion, extension identity, identity, and negation are also introduced by definition:

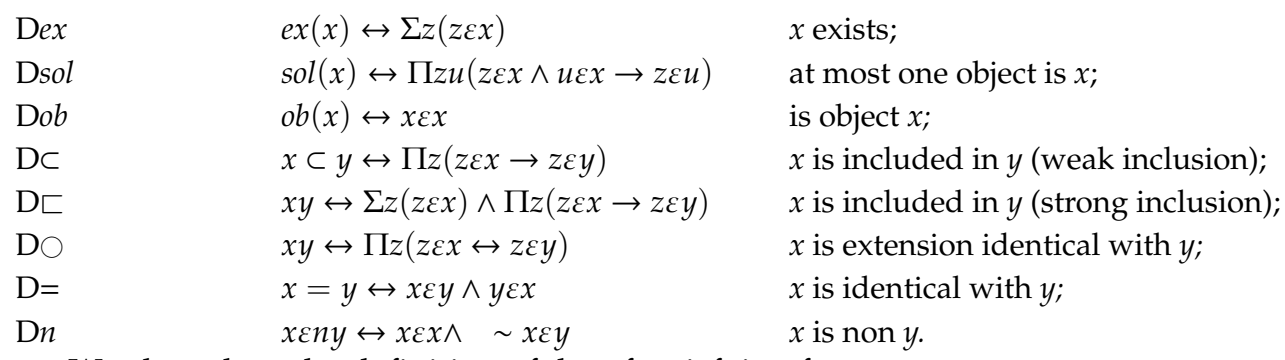

We also adopt the definition of the of satisfying functor:

$$
\text { Dsts } f x \varepsilon s t s f(\phi) \leftrightarrow x \varepsilon x \wedge \phi(x) \quad x \text { is satisfying } \phi
$$

The system of elementary ontology can be founded on the calculus of predicates without identity. Jerzy Słupecki's work [1] may serve as an introduction to elementary ontology.

\subsection{Peano's Axiomatics}

The system of arithmetic of natural numbers can be founded on the axiomatics given by Peano [2]. The formulation of these axiomatics in the framework of elementary ontology can be found in Leśniewski's works ([3], chapter 4, p. 129):

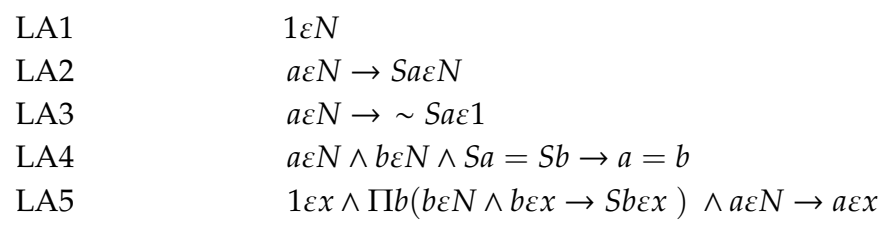

The last axiom is theaxiom of mathematical induction. Axioms A1-A5, as depicted below in Section 4, are equivalent to the axioms given by Peano.

\subsection{Elementary Ontology with Frege's Predication Scheme}

Here, the system of elementary ontology is extended with the functor sub of then $/ n$ category. The elementary expression " $x \varepsilon s u b(y)$ " is read here as: " $x$ is subordinated to $y$ ". The specific axioms of this system $\left(\mathbf{O E}^{\text {sub }}\right)$ have the following shapes (see [4]):

SA1

SA2

SA3

SA4

$$
\begin{aligned}
& x \varepsilon s u b(y) \rightarrow \operatorname{sub}(x) \subset \operatorname{sub}(y) \\
& x \varepsilon s u b(y) \rightarrow \sim y \subset \operatorname{sub}(x) \\
& x \varepsilon s u b(y) \rightarrow y \varepsilon y \\
& \operatorname{sub}(x) \operatorname{sub}(y) \rightarrow y y
\end{aligned}
$$

The functor of concept is introduced by definition:

DC $\quad x \varepsilon C y \leftrightarrow x \varepsilon x \wedge \Pi z(z \varepsilon y \leftrightarrow z \varepsilon s u b(x)) \quad x$ is a concept $y$

The key theses with functors $s u b$ and $C$ include:

$x \varepsilon s u b(C y) \rightarrow x \varepsilon y \quad$ If $x$ is subordinated to the concept $y$, then $x$ is $y$,

and:

$C x \varepsilon s u b(C y) \rightarrow x \subset y \quad$ If the concept $x$ is subordinated to the concept $y$, then $x$ is included in $y$ 


\section{Idea}

The term "Anzahl" functions in German in various numerical phrases, more or less defined. The word Anzahl appears in such contexts as: Anzahl der Äpfel (number of apples), Anzahl von Freuden (number of friends), fünf is Anzahl der Bäumen in meinem Garten (five is the number of trees in my garden), or neun ist Anzahl der Planeten des Sonnensystems (nine is the number of planets of the Solar System).

\subsection{An Indefinite Numerical Phrase with the Term Anzahl}

In number phrases of the indefinite type we have to deal with the combination of the number name (Zahl) being an abstract object with the general name, which is the product of a general name (e.g., apple/apples) and a name present only implicitly, which somehow characterizes the objects falling within the extension of the first name (usually a general name referring to the place they occupy). For example, "five apples" occupy a certain place on the table.

A phrase of this type, which is a nominal expression, can be rendered as follows:

$$
a \circ x
$$

where $a$ is a number (Anzahl) and $x$ is the name of object of a given sort, which, in combination with a hidden name characterizing these objects, gives us our universe of discourse. Since $a$ and $x$ are names, $a \circ x$ is also a name, then $\circ$ is a functor of the $-n / n n$ category.

\subsection{A Definite Numerical Phrase with the Term Anzahl}

Numerical phrases of the definite type ("neun ist Anzahl den Planeten des Sonnensystem" or "Anzahl... ist gleich dem Anzahl...") are discussed in Gottlob Frege's works (see [5], p. 20 and [6], p. 88). In number phrases of the definite type, we use an elementary expression of the type:

$$
a \varepsilon A x
$$

where a is a number, $\varepsilon$ is a functor (of the $s / n n$ category), and $x$ is a (definite) general name. A specific functor $A$ (from Anzahl) of the $n / n$ category appears here.

In Frege, "Anzahl" refers to a concept designated by a single-argument predicate (Begriffswort), but also a functorof the $n /(s / n)$ category. The elementary phrase with this functor could be expressed in the following way: $a \varepsilon A(P)$, where the (single-argument) predicate $\mathrm{P}$ is equivalent to the name $\mathrm{x}$ (from the phrase " $a \varepsilon A x^{\prime \prime}$ ). Our expression existing in the framework of the calculus of names is closer to natural language, in which the category of names is understood broadly (individual and general names), as opposed to only individual names, as in Frege's language (Namen=Eigennamen).

We will continue to deal with definite number phrases with the term Anzahl. We shall return to indefinite phrases with this term in the final part of the present paper.

\section{Arithmetic of Natural Numbers}

As our starting point, we shall adopt the following five axioms given by Leśniewski, including 0 in natural numbers, which is in accordance with the contemporary formulations of Peano's axiomatics:

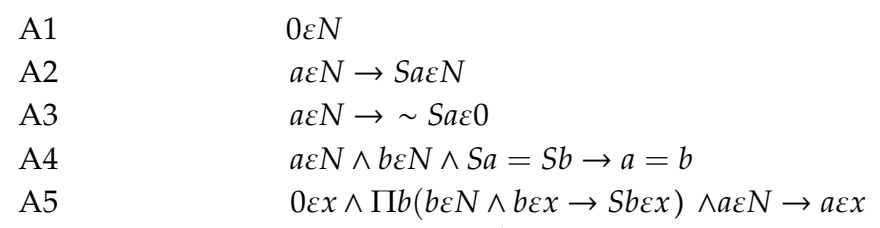

Our initial system is $\mathbf{O E}^{s u b}$ enriched with these axiomatics. The language of this system $\left(\mathrm{OE}^{\text {sub }}[\mathrm{A} 1, \mathrm{~A} 2, \mathrm{~A} 3, \mathrm{~A} 4, \mathrm{~A} 5]\right)$ is extended with number nominal variables $(a, b, c)$, referring to natural numbers. Apart from the standard rule of detachment (MP): 
$\mathrm{MP} \quad \alpha, \alpha \rightarrow \beta / \beta$

we shall adopt the rule of substitution:

RS $\quad \alpha / \alpha[x / t]$,

where $t$ is a nominal variable/constant or (in particular) a number nominal variable/constant:

$\alpha / \alpha[a / t]$,

where $t$ is a number nominal variable/constant.

Having adopted the definition of the natural number:

$\mathrm{DN} \quad a \varepsilon N \leftrightarrow a \varepsilon a \wedge \Sigma x(a \varepsilon A x)$,

we can eliminate constant $N$ by means of functor $A$ :

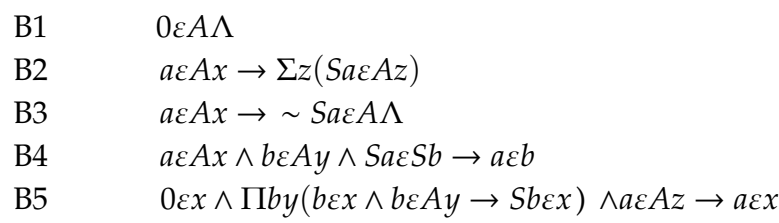

Axioms A1-A5 are consequences of Axioms B1-B5:
(A1)
$0 \varepsilon N$
$a \varepsilon N \rightarrow$ SacN
$a \varepsilon N \rightarrow \sim \operatorname{Sac} 0$
$a \varepsilon N \wedge b \varepsilon N \wedge S a=S b \rightarrow a=b$
$[\mathrm{B} 1, \mathrm{DN}]$
$[\mathrm{DN}, \mathrm{B} 2]$
$[\mathrm{DN}, \mathrm{B} 1, \mathrm{R} 2, \mathrm{~B} 3]$
(A4)
$[\mathrm{DN}, \mathrm{D}=, \mathrm{B} 4]$
(A5)
$0 \varepsilon x \wedge \Pi b(b \varepsilon N \wedge b \varepsilon x \rightarrow S b \varepsilon x) \wedge a \varepsilon N \rightarrow a \varepsilon x$
[DN,B5]

Next, we shall eliminate from axiomatics the functor of the successor $(S)$ by introducing it by definition. We shall leave it only in the last axiom, for the purpose of shortening.

We shall adopt the axiomatics:

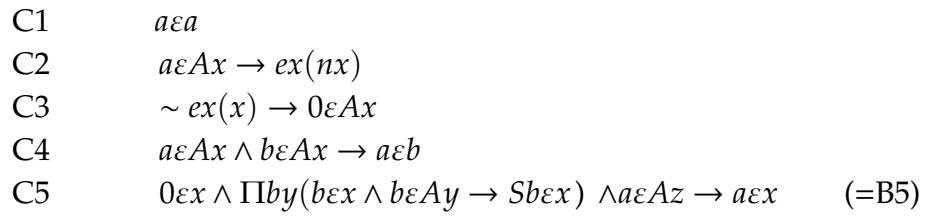

We shall introduce the functor of the successor by definition:

DS $a \varepsilon S b \leftrightarrow a \varepsilon n b \wedge \prod x z(b \varepsilon A x \wedge z \varepsilon n x \leftrightarrow a \varepsilon A(x \cup z) \wedge z \varepsilon n x) \wedge \prod x z(0 \varepsilon A x \wedge z \varepsilon n x \rightarrow a \varepsilon A z) \wedge$ $\Pi y z(a \varepsilon A z \wedge \sim \operatorname{ex}(z) \rightarrow \sim b \varepsilon A y) \wedge \Pi z(a \varepsilon A z \wedge z \varepsilon z \rightarrow b=0)$

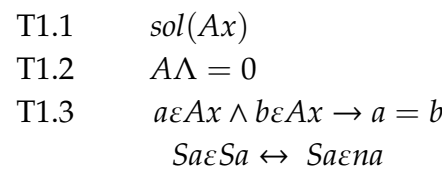

$\mathrm{T} 1.4$

$\mathrm{T} 1.5$

$\mathrm{T} 1.6$

$\mathrm{T} 1.7$

$\mathrm{T} 2.1$

T2.2

T2.3

$\mathrm{T} 2.4$

$\mathrm{T} 2.5$

$$
\begin{aligned}
& \operatorname{Sac} A(x \cup z) \wedge z \varepsilon n x \rightarrow a \varepsilon A x \\
& \operatorname{Sa\varepsilon Ax} \wedge x \varepsilon x \rightarrow a=0 \\
& \sim \text { Saca } \\
& 0 \varepsilon A \Lambda \quad \text { (=B1) } \\
& a \varepsilon A x \rightarrow \Sigma z(\operatorname{Sac} A) \\
& \text { (=B2) } \\
& a \varepsilon A x \rightarrow \sim \text { SacA } \Lambda \\
& \text { (=B3) } \\
& a \varepsilon A x \wedge b \varepsilon A y \wedge S a \varepsilon S b \rightarrow a \varepsilon b \\
& \text { (=B4) }
\end{aligned}
$$$$
\wedge \sim \operatorname{ex}(z) \rightarrow \sim a \varepsilon A y) \wedge \prod z(\operatorname{Sa\varepsilon } A z \wedge z \varepsilon z \rightarrow a=0)
$$$$
\wedge \Pi x z(a \varepsilon A x \wedge z \varepsilon n x
$$$$
\leftrightarrow \operatorname{Sac} A(x \cup z) \wedge z \varepsilon n x)
$$$$
\wedge \prod x z(0 \varepsilon A x \wedge z \varepsilon n x \rightarrow S a \varepsilon A z)
$$$$
\wedge \Pi y z(\operatorname{Sa\varepsilon Az}
$$$$
\phi(0) \wedge \Pi b(b \varepsilon N \wedge \phi(b) \phi(S b)) \wedge a \varepsilon N \rightarrow \phi(a)
$$

[C4,Dsol] $[\mathrm{OE}, \mathrm{C} 3, \mathrm{~T} 1.1, \mathrm{R} 3, \mathrm{D}=]$

$[\mathrm{C} 4, \mathrm{D}=]$

[DS]

[R1,T1.4]

[R1,T1.4]

[R1,T1.4.Dn]

[OE,C3]

$[\mathrm{C} 2, \mathrm{C} 1, \mathrm{Dex}, \mathrm{T} 1.4]$

[R1,T1.4,OE]

[OE,T1.4,C2,Dex,T1.5,T1.3]

[C1,Dstsf,DN,C5] 
Of these, T2.5 is a typical formulation of an induction axiom.

\section{Addition and Multiplication: Logical and Philosophical Analysis}

Addition and multiplication as operations on numbers-with the numerical functor (Anzahl) as a primary functor-is, from a logical and philosophical point of view, an operation more complex than it usually appears.

\subsection{Addition}

The addition of numbers in the context of a numerical functor (Anzahl) establishes the number of objects of the same sort. For example, withfive trees in the front garden and seven trees in the back garden, we reach the conclusion that we have 12 trees altogether. Using names $y$ and $z$ so that ya tree in the front garden and za tree in the back garden, and taking into account the fact that the extensions of these names have no common elements $(\sim \operatorname{ex}(y \cap z))$, we create in this case a common name $x a$ tree in the garden, so that $x y \cup z$-in order to state the following: $12 \varepsilon A x$ with $5 \varepsilon A y$ and $7 \varepsilon A z$, where between $x, y$, and $z$ there are the connections described before. The operation of addition in this context can be generally defined as follows:

$$
\mathrm{D}+a \varepsilon b+c \leftrightarrow a \varepsilon a \wedge \Sigma x y z(x y \cup z \wedge \sim \operatorname{ex}(y \cap z) \wedge a \varepsilon A x \wedge b \varepsilon A y \wedge c \varepsilon A z)
$$

\subsection{Multiplication}

The multiplication of numbers in the context of the numerical functor (Anzahl) is more complicated. Let us take a similar example. Let us assume that in the garden we have trees which are grouped in four groups, each comprising five trees. There are two groups in front of the house (on the left and on the right) and two groups behind the house (one on the left and one on the right, too). We beginour operation by distinguishing four groups, which we treat as distributive classes determined by four names: $y, z, u$, and $v$, which are, respectively a tree in the front garden on the left, a tree in the front garden on the right, a tree in the back garden on the left, and a tree in the back garden on the right.

We shall mark these classes, respectively, as: $C y, C z, C u$, and $C v$. This distinction is accom- panied by an ascertainment that for a certain name $x$ with the extension- $x y \cup z \cup u \cup v(x a$ tree in the garden), these names are separate in terms of extension $\sim \operatorname{ex}(y \cap z), \sim \operatorname{ex}(z \cap u)$, and $\sim \operatorname{ex}(u \cap v)$. Next, we state that there are four such groups/classes of trees-4cAw $4 \varepsilon A w$, where $w$ is a shortening of the name "a group of trees in the garden", and we finally state that each group of trees comprises five trees and each tree in the garden can be characterized as belonging strictly to one of these groups and as one of five trees belonging to this group. This last sentence can be shortly expressed as follows: each tree in the garden is characterized by a pair [one of the groups, one of the trees of a given bank of trees assigned to this group], to be eventually able to say that the number of trees in the garden is identical to the number of such pairs. Symbolically, it can be briefly expressed as follows:

$$
\mathrm{D} \bigodot a \varepsilon b \bigodot c \leftrightarrow a \varepsilon a \wedge \Sigma x y z(a \varepsilon A x \wedge b \varepsilon A y \wedge c \varepsilon A z \wedge \Pi u(u \varepsilon y \leftrightarrow \Sigma v(u \varepsilon C v \wedge v \subset x)) \wedge x \varepsilon y \times z)
$$

where $\times$ is a functor of Cartesian product of the $n / n n$ category.

\section{The New Formulation of the Arithmetic of Natural Numbers}

We shall replace axiom C3 with a more intuitive one:

$$
\mathrm{C}^{*} \quad x y \rightarrow A x A y
$$

The system of arithmetic of natural numbers in this formulation is: $\mathrm{OE}^{\text {sub }}\left[\mathrm{C} 1, \mathrm{C} 2, \mathrm{C} 3{ }^{*}, \mathrm{C} 4, \mathrm{C} 5\right]$. In the formulation of axiom C5, the functor of successor occurs, which we are introducing similarly, by means of the already given definition $\mathrm{DS}$.

Here, the term $C$ from $\mathbf{O E}^{s u b}$ is interpreted in terms of class (see [7]): 
DC $\quad x \varepsilon C y \leftrightarrow x \varepsilon x \wedge \Pi z(z \varepsilon y \leftrightarrow z \varepsilon s u b(x)) \quad x$ is a distributive class (of objects which are) $y$.

In addition to the rules of substitution and detachment (MP), we shall also adopt the rules of omission and introduction for the list operator in the form (see [8]):

OL $\quad x \varepsilon\left[z_{1}, \ldots, z_{n}\right] / x \varepsilon z_{1} \vee \ldots \vee x \varepsilon z_{n} \quad\left[x_{1}, \ldots, x_{n}\right] \varepsilon y / x_{1} \varepsilon y \wedge \ldots \wedge z_{n} \varepsilon y \quad$ if $y$ is not list

IL $\quad x \varepsilon z_{1} \vee \ldots \vee x \varepsilon z_{n} / x \varepsilon\left[z_{1}, \ldots, z_{n}\right] \quad x_{1} \varepsilon y \wedge \ldots \wedge z_{n} \varepsilon y /\left[x_{1}, \ldots, x_{n}\right] \varepsilon y$

and the rule:

$\mathrm{RL} \quad\left[x_{1}, \ldots, x_{n}\right] \varepsilon\left[z_{1}, \ldots, z_{n}\right] / x_{1} \varepsilon z_{1} \wedge\left[x_{2}, \ldots, x_{n}\right] \varepsilon\left[z_{2}, \ldots, z_{n}\right] \quad[x] \varepsilon[z] / x \varepsilon z$

Thanks to this rule, we obtain the property for two-element lists, $[x, y]=[z, u] \rightarrow x=z \wedge y=u$, which is the equivalent of property for an orderly pair in the framework of the set theory.

Now, let us define the functor of Cartesian product (compare [9], p. 176):

$\mathrm{D} \times \quad x \varepsilon y \times z \leftrightarrow x \varepsilon x \wedge \Pi u v w(u \varepsilon[u, w] \wedge v \varepsilon y \wedge w \varepsilon z \leftrightarrow u \varepsilon s u b(x))$

Now, we can-quite formally-adopt the definitions of the operations of addition $(+)$ and multiplication $(\odot)$ in accordance with their interpretation:

$\mathrm{D}+\quad a \varepsilon b+c \leftrightarrow a \varepsilon a \wedge \Sigma x y z(x y \cup z \wedge \sim e x(y \cap z) \wedge a \varepsilon A x \wedge b \varepsilon A y \wedge c \varepsilon A z)$

$\mathrm{D} \odot a \varepsilon b \bigodot c \leftrightarrow a \varepsilon a \wedge \Sigma x y z(a \varepsilon A x \wedge b \varepsilon A y \wedge c \varepsilon A z \wedge \Pi u(u \varepsilon y \leftrightarrow \Sigma v(u \varepsilon C v \wedge v \subset x)) \wedge x \varepsilon y \times z)$

According to $\mathrm{D}+$, addition is a symmetrical operation: $a \varepsilon b+c \leftrightarrow c+b(a+b=b+a)$.

The operation of multiplication in the sense $\odot$, in accordance with the definition $\mathrm{D} \odot$, is not symmetrical. We shall introduce multiplication as a symmetrical operation $(\cdot)$ by definition:

D. $\quad a \varepsilon b \cdot c \leftrightarrow(a \varepsilon b \bigodot c) \vee a \varepsilon c \bigodot b)$

The theses which are consequences of definition D0 and axiom A3* include:

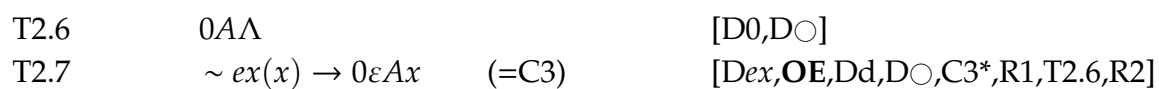

\section{The Term Anzahl in the Indefinite Sense}

We shall now deal with number phrases with the term "Anzahl" in the indefinite sense, where, "Numbers (Anzahlen) are always numbers (Anzahlen) of something. They can be added (five apples and two apples make seven apples). However, it is impossible to multiply numbers (Anzahlen) by numbers (Anzahlen)" (see [10], p. 7).

Compounds such as five apples or two dogs fall, in accordance with the previous arrangements, under the scheme $a \circ x$. We shall introduce the functor $\circ$ by definition:

Do $x \varepsilon a \circ y \leftrightarrow x \varepsilon x \wedge \Sigma z(a \varepsilon A(y \cap z))$

The numbers (Anzahlen) in this sense behave like the so-called denominate numbers (like, for example: $5 \mathrm{~m}$ or $2 \mathrm{~kg}$ ) present in the so-called dimensional analysis, which appears in physics. They can be added, provided that the same unit is preserved. Addition in such contexts can be defined as follows:

$$
\mathrm{D} \bigoplus x \varepsilon(a \circ y) \bigoplus(b \circ y) \leftrightarrow x \varepsilon(a+b) \circ y
$$

Denominate numbers (benannte Zahlen) are the subject of analysis in one of Hermann von Helmholtz's works (see [11], p. 12).

The numbers (Anzahlen) in the indefinite sense cannot be multiplied, as opposed to denominate numbers in dimensional analysis. 


\section{Conclusions}

A new, original depiction of arithmetic of natural numbers with Anzahl as a primitive term has been presented. The basis of this depiction is the calculus of names, which is a certain extension of elementary ontology $\left(\mathbf{O E}^{\text {sub }}\right)$. The notion of a pair (list) was introduced by means of rules OL, IL, and RL, the last of them playing a significant role. I have recently noticed that it is possible to define an ordered pair in the framework of the $\mathbf{O E}^{\text {sub }}$ system.

Funding: This research received no external funding.

Acknowledgments: I would like to express my sincere gratitude and highest appreciation to all referees of this article for all their useful remarks, comments, and suggestions to the content of the article and also its Englishlanguage verification. Individual thanks are due, in particular, to Luna Shen and Hunter Jia for their precious support.

Conflicts of Interest: The authors declare no conflict of interest.

\section{References}

1. Słupecki, J.S. “Leśniewski Calculus of names”. Studia Log. Int. J. Symb. Log. 1955, 3, 7-70.

2. Peano, G. Arithmetices Principia Nova Methodo Exposito; Bocca: Turin, Italy, 1889.

3. Leśniewski, S. Lecture Notes in Logic; Srzednicki, J.T.J., Stachniak, Z., Eds.; Kluwer Academic: Dordrecht, The Netherlands, 1988.

4. Wojciechowski, E. "Rachunek nazw i schemat predykacji z Begriffschrift Gottloba Fregego" (Calculus of Names and Predication Scheme from Gottlob Frege's Begriffsschrift). In Predykacja, negacja i kwantyfikacja (Predication, Negation and Quantification); Wojciechowski, E., Ed.; Aureus: Kraków, Poland, 2019.

5. Frege, G. Die Grundlagen der Arithmetik: Eine Logisch Mathematische Untersuchung über den Begriff der Zahl; Verlag von Wilhelm Koebner: Breslau, Germany, 1884.

6. Patzig, G. Gottlob Frege und die logische Analyse der Sprache. In Sprache Und Logik, 2nd ed.; Patzig, G., Ed.; Vandenhoeck \& Ruprecht: Göttingen, Germany, 1981; pp. 77-100.

7. Wojciechowski, E. "Klasy dystrybutywne i klasy kolektywne” (Distributive Classes and Collective Classes). In Predykacja, negacja i kwantyfikacja (Predication, Negation and Quantification); Wojciechowski, E., Ed.; Aureus: Kraków, Poland, 2019.

8. Wojciechowski, E. "Rachunek nazw z listami” (The Calculus of Names with Lists). Rocz. Filoz. 2011, 59, 35-50.

9. Borkowski, L. Logika Formalna (Formal Logic), 2nd ed.; PWN: Warszawa, Poland, 1977.

10. Grote, A. Anzahl, Zahl und Menge. Die Phänomenologischen Grundlagen der Arithemitk; Felix Meiner Verlag: Hamburg, Germany, 1983.

11. Von Helmholz, H. Zählen und Messen, erkenntnisstheoretisch betrachtet. In Philosophische Aufsätze, Eduard Zeller Ze Seinem Fünfzigjährigen Doctorjubiläum Gewidmet; Fues Verlag: Leipzig, Germany, 1887; pp. 17-52.

(C) 2019 by the author. Licensee MDPI, Basel, Switzerland. This article is an open access article distributed under the terms and conditions of the Creative Commons Attribution (CC BY) license (http://creativecommons.org/licenses/by/4.0/). 